\title{
ViVan Formula- A Modified Formula for Estimating the Mesiodistal Width of Maxillary Central and Lateral Incisor
}

\author{
Vikrant V. Jadhav ${ }^{1}$, Vasudevan S.D. ${ }^{2}$, Ranjit Kamble ${ }^{3}$, Meenakshi M. Tiwari ${ }^{4}$
}

1Department of Orthodontics and Dentofacial Orthopedics, Sharad Pawar Dental College and Hospital, Sawangi, Wardha, Maharashtra, India. ${ }^{2}$ Department of Orthodontics and Dentofacial Orthopedics, Sharad Pawar Dental College and Hospital, Sawangi, Wardha, Maharashtra, India. ${ }^{3}$ Department of Orthodontics and Dentofacial Orthopedics, Sharad Pawar Dental College and Hospital, Sawangi, Wardha, Maharashtra, India. ${ }^{4}$ Department of Orthodontics and Dentofacial Orthopedics, Sharad Pawar Dental College and Hospital, Sawangi, Wardha, Maharashtra, India.

\section{ABSTRACT}

\section{BACKGROUND}

It is a frequent occurrence that teeth especially maxillary incisors are either completely avulsed or fractured due to trauma in facial region. Replacement of these missing teeth always possess a challenge if there are no prior dental records of the patient. Also, maxillary lateral incisors are frequently smaller in size - "Peg shaped" or congenitally absent. Aesthetic correction or/and replacement of this tooth also poses a problem when prior records are missing.

\section{METHODS}

Totally, 300 study casts were taken from the patients and digital vernier caliper was used to measure the mesiodistal width of maxillary central and lateral incisors and mandibular lateral incisors on both the sides. The mean and the standard deviations of the width was assessed. Similarly, the proportion between the widths of the maxillary lateral incisor and mandibular lateral incisor was also assessed and based on the proportions, the ViVan formula was derived.

\section{RESULTS}

Based on the proportions, ViVan Formula for Central Incisor and Lateral Incisor is derived.

\section{CONCLUSIONS}

ViVan ratio determined the mesiodistal width of central and lateral incisor with the help of mandibular lateral incisor. This formula provides results accurately and with minimum error. One of the important diagnostic tools is the use of diagnostic model for estimation of tooth size. Tooth size must be proportionate to jaw size so as to avoid tooth size arch length discrepancy.

\section{KEY WORDS}

Central Incisor, Lateral Incisor, Missing Teeth, Mesiodistal Width
Corresponding Author: Dr. Vikrant V. Jadhav, Junior Resident,

Department of Orothodontics and Dentofacial Orthopedics, SPDC,

Sawangi, Wardha, Maharashtra, India. E-mail: vikrantjadhav9405@gmail.com

DOI: $10.14260 /$ jemds/2020/248

Financial or Other Competing Interests: Dr. Jadhav has a patent copyright licensed.

How to Cite This Article:

Jadhav VV, Vasudevan SD, Kamble R, et al. ViVan Formula- A modified formula for estimating the mesiodistal width of maxillary central and lateral incisor. J. Evolution Med. Dent. Sci. 2020;9(14): 1144-1147, DOI:
Submission 07-01-2020, Peer Review 14-03-2020, Acceptance 20-03-2020, Published 06-04-2020. 


\section{BACKGROUND}

For establishing and maintaining the good and well accepted occlusion is the ultimate aim of any orthodontic treatment. The knowledge which is available to us on mesiodistal width on tooth size is limited. It is observed that proper tooth dimensional relationship is necessary to establish good interdigitation. It is necessary to determine the location and amount of a tooth size disproportion before initiating the orthodontic treatment. This is because these conditions will often lead to either removal or addition of tooth structure to open or close spaces in the opposing arches. Mesiodistal width of tooth is recognised as a primitive etiologic factor in space anomalies, which composed with tooth width disproportion may lead to malocclusion.

One of the basic concept or clinical situations which orthodontist faces in day-to-day life is manage space discrepancy. Tooth size ratios which are available have many limitations and needs certain diagnostic setup. Complex cases are difficult to manage with available formulas for determination of tooth size. Enough data is available as evidence that population differ w.r.t. inter-arch tooth size relationships. This is because tooth sizes are not systematic.

There are different methods to determine the width of missing teeth. Tonn's(1) developed a method to evaluate the width of missing maxillary lateral incisor. This formula required the width of all erupted incisors (maxillary and mandibular) to calculate the width of missing maxillary lateral incisor. Hence, it could not be applied if any teeth were missing. It was also time consuming. Furthermore, this formula was derived for the Caucasian population. So, many authors questioned its applicability in Indian population.(2-7) when the formula was applied in our population, inaccurate results were obtained. To overcome these problems, "ViVan formula" for determining mesiodistal width of missing teeth based on the width of mandibular lateral incisor for our population were derived.

The new formula is simple to use, easy to remember and does not involve all incisors for calculation. Therefore, this formula is easy to apply in any conditions. Permanent mandibular Lateral are mostly not missing or congenitally absent. Therefore, these teeth were given the preference. The formula was applied and checked in our population.

It would benefit the patients seeking aesthetic correction for replacement of missing permanent maxillary central and/ lateral incisors. The formula can also be applied in cases of aesthetic correction of malformed/microdontia of permanent maxillary lateral incisors. This could be used by all the branches of dentistry. This formula will help us to achieve aesthetic profile and proper occlusion.

\section{METHODS}

The present study was carried out at after the approval from the Institutional Ethical committee. For the study, the potential participant was examined clinically. The following inclusion and exclusion criteria were used to select the participants.

\section{Inclusion Criteria}

1. Participants in OPD with all permanent teeth fully erupted with or without third molar.

2. Participants with age 18-30 with Angles Class I canine and molar relation.

\section{Exclusion Criteria}

Participants with

1. Multiple missing teeth.

2. Unerupted permanent teeth

3. Unrestored carious teeth.

4. Teeth with full coverage restoration.

5. Proximal wear/attrition of teeth.

6. Congenital defects or deformed teeth.

Informed consent was obtained from those participants who meet the inclusion criteria. A total of 300 participants were included. Following this, accurate impression of upper and lower dental arches was recorded using Putty impression material. The impression was poured immediately with dental stone (Kaldent Dental Plaster Class III) to minimize distortion of impression. The cast were retrieved after the dental stone was set and bases were poured, and measurement were carried out on the casts.

A modified digital vernier caliper was used to measure the mesiodistal width of the teeth. The tips of the caliper were modified to fit into the contact points of the teeth. The tips of the caliper were placed in the contact point of the tooth and the mesiodistal width of the teeth was recorded and entered into the table shown below. All the measurements were carried out in millimeters. (Table no. 1)

The mesiodistal width of maxillary central and lateral incisors and mandibular lateral incisors on both the sides were measured using digital vernier caliper on 300 study cast. The mean and the standard deviations of the width was assessed. The proportion between the widths of the maxillary central incisor and mandibular lateral incisor was assessed. Similarly, the proportion between the widths of the maxillary lateral incisor and mandibular lateral incisor was also assessed and based on the proportions, the ViVan formula was derived.). This study is observation study. The sample size was derived by complete enumeration method.

This formula is derived with the help of mesiodistal width of permanent mandibular lateral incisor. Lateral incisor is selected for deriving the mesiodistal width if teeth because it has less prevalence of congenitally missing and undergoing extractions. With various permutation and combination method this formula was derived to provide accurate results with least errors.

\section{RESULTS}

Based on the Proportions, ViVan Formula for Central Incisor

$\frac{\text { Mesiodistal Width of Lower Lateral Incisor X } 4}{3}$ $-0.1$

When this formula was checked in our population, we obtained following results- 
For maxillary central incisor, to calculate with, mean of mandibular lateral incisor and maxillary central incisor was calculated. It was found to be: minimum 0.55 and maximum 0.85. Mean standard error was calculated that was 0.004 on left side and 0.006 on right side. Standard deviation of 0.056 , Average Difference (error) of 0.04 in the right quadrant means right maxillary central incisor and in Error of 0.06 in the left quadrant means left maxillary central incisor was obtained. Co-efficient of variance was found to be 0.03 for right side and for left side it was 0.03 . Based on these mathematical values, it was possible to determine the accurate formula with minimum error for our population. The mesiodistal width of permanent maxillary central incisor using mesiodistal width of lower lateral incisor. Error was obtained similar on both the sides of arch. The error was very negligible. For more accurate results \pm 0.5 to be done in results.

Comparison of sizes between maxillary lateral incisor and mandibular lateral incisor,

\section{Based on Proportions, ViVan Formula for Lateral Incisor}

\section{Mesiodistal Width of Lower Lateral Incisor X 4} 3

Similarly, for maxillary Lateral incisor, mean of mandibular lateral incisor and maxillary lateral incisor was calculated. It was found to be: minimum 0.55 and maximum 0.92. Mean standard error was calculated that was 0.004 on left side and 0.007 on right side. Standard deviation of 0.824 , Average Difference (error) of 0.03 in the right quadrant means right maxillary central incisor and in Error of 0.06 in the left quadrant means left maxillary lateral incisor was obtained. Co-efficient of variance was found to be 0.03 for right side and for left side it was 0.06. Based on these mathematical values, it was possible to determine the accurate formula with minimum error for our population which could be used to determine the mesiodistal width of permanent maxillary lateral incisor using mesiodistal width of lower lateral incisor. Error was obtained similar on both the sides of arch. The error was very negligible. For more accurate results \pm 0.5 to be done in results.

Based on the obtained results final formulas which were derived are -

\section{ViVan Formula for Maxillary Central Incisor}

Mesiodistal Width of Mandibular Central Incisor X 4 3

Mesiodistal Width of Mandibular Central Incisor X 4 3

\section{DISCUSSION}

Esthetics has a vital part in Orthodontics. Goal of an Orthodontist is to create a structural balance, functional efficiency and esthetic harmony. Major considerations are given for creating harmonious smile. To achieve this goal, a harmonious smile, the Orthodontist needs to plan these cases with carefully. Otherwise, managing or space closure becomes a challenge for Orthodontist. Proper determination of tooth size and decision for managing the cases with missing anteriors becomes crucial. These cases are difficult to treat without proper analysis available. In these circumstances, Orthodontist must appropriately select the dimensions for tooth size evaluation. But, till date these are no such analysis which gave accurate results without any errors in result. Therefore, selection of appropriate dimension becomes difficult. Orthodontist must plan judiciously before treating cases with missing anteriors. Other than managing tooth dimension (tooth size) it is important to manage tooth form and colour. This all criteria depends on proper tooth size. This require both ability and methodical information. This will be brought by sound knowledge of formulas available to us. For this ViVan formula is developed. Mostly, patients are concerned with anterior teeth for esthetic purpose. Therefore, suitable formula is provided to achieve appropriate results.

In the era of 19th century, mostly procedures involved "hunt and peck" or "trial and error" till dentist was content with precise tooth size. Implemented systematic and scientific approach is required while treating these situations. It is clear that to appear attractive, maxillary anterior teeth should be in appropriate proportion to facial morphology. ${ }^{(8)}$

Enrichment of facial appearance is one of the reasons for patients receiving orthodontic treatment. Missing teeth, specifically maxillary anterior teeth experience trauma frequently. So, mostly they are missing. Orthodontic treatment should fulfill the esthetic demands. Achieving perfect tooth size harmony during orthodontic treatment is difficult. Achieving best result with minimum treatment duration and perfectness is necessary for orthodontic treatment. This is most challenging tasks in orthodontics.

During Orthodontic treatment, when no pre-extraction records are available, determining accurate dimension of tooth size and shape are difficult. Especially when the teeth are missing in anterior region. A systematic method is required during selection of tooth size.

Missing central incisors are often encountered in clinical practice. This is seen in patients who have had traumatically avulsed central incisors. According to epidemiological studies it is $19.8 \%{ }^{(8)}$ Agenesis of maxillary lateral incisors are also often encountered, it about $23 \%$ of the population. ${ }^{(8)}$ According to epidemiological studies, one or both of the maxillary incisors are congenitally missing in about $12 \%$ of the population.(8) Maxillary lateral incisors are the third most common missing teeth after third molars and mandibular second premolars. ${ }^{(8)}$

Missing maxillary incisor(s) have a psychological implication due to deprived smile, aesthetics and improper 0.72 speech.(9) Thus, these teeth are indicated for replacement. This problem is encountered in deciduous dentition.(10),(11) In order to achieve an optimum aesthetic and functional result, it is often necessary to establish a synchronised, interdisciplinary approach with orthodontist to know correct mesiodistal width of teeth. It is difficult to estimate the widths of the missing incisors when previous dental records of the patients are missing. This is more so in our set up as patients rarely visit dentists at regular intervals. 
The incidence of congenital absence or missing/malformed/impacted/unerupted incisors though is high. This is the area of concern for dentist in general and orthodontists in particular. Also, chances of losing anteriors due to periodontal pathology or trauma or congenital reason are high due to its location. Using the concept of proportionality that exists between the teeth of maxillary and mandibular teeth, Tonn's(1) in 1937 gave the formula to determine the width of missing / unerupted / malformed / unerupted central incisor and lateral incisor. The formula was difficult to be used as all the teeth in the oral cavity of the patient had to be measured. Also, its application was limited as it was population specific. Hence, the ViVan formula was developed. The formula was derived by analysing the dental study models of 300 individuals.

The mesiodistal width of the maxillary and mandibular permanent incisors were measured using modified digital vernier calliper and tabulated. The mean and standard deviation were calculated. The dimensions of the maxillary and mandibular teeth were then compared. Based on the theory of proportions, the formula for calculating dimensions of the maxillary permanent central and lateral incisors were based on the width of mandibular lateral incisor. Average difference between the measured and estimated values of maxillary left lateral incisor was $0.06 \mathrm{~mm}$ when mandibular left lateral incisor was used. For the maxillary left central incisor the error was $0.03 \mathrm{~mm}$. Similarly, a negligible error was observed when the mandibular right lateral incisor was used to predict the widths of the left maxillary central and lateral incisors. Also, the difference was in the second decimal when the mandibular incisor of same and contra-lateral sides was used to predict the widths of maxillary right central and lateral incisors.

Therefore, there was a need to derive a formula to estimate the widths of incisors from the other teeth. Mandibular lateral incisors are rarely missing. Furthermore, they rarely show any deformity. This could be used as a guide to replace the missing teeth. Their measurement is also easy. So, ViVan formula was derived to estimate the widths of central and lateral incisors for our population.

The formula that we propose is as follows-

\section{ViVan Formula for Upper Lateral Incisor}

$\frac{\text { Mesiodistal Width of Lower Lateral Incisor X 4 }}{3}-0.7$
$\frac{\text { Mesiodistal Width of Lower Lateral Incisor X 4 }}{3}-0.1$

\section{CONCLUSIONS}

The success of orthodontic treatment depends on careful approach of record taking and diagnosis. One of the important diagnostic tools is the use of diagnostic model for estimation of tooth size. Tooth size must be proportionate to jaw size so as to avoid tooth size arch length discrepancy. For this, a simple mathematical formula (ViVan's Formula) can be used as a valuable clinical aid in predicting an approximate value for the tooth. The accuracy of these formulas was checked in our population and significant results were obtained.

\section{REFERENCES}

[1] Tonn P. Uber die mesio-distalen Zahn breiten relationen der Zahne des oberkiefers zudenents prechenden des unter kiefersbei normalen and anormalen occlusion. Diss. Berlin 1937.

[2] Bolton WA. Disharmony in tooth size and its relation to the analysis and treatment of malocclusion. Angle Orthod 1958;28:113-30.

[3] Jindal R, Bunger E. Bolton's intermaxillary tooth size ratios among school going children in Punjab population. Indian Journal of Oral Sciences 2013;4(3):110-3.

[4] Subbarao VV, Regalla RR, Santi V, et al. Interarch tooth size relationship of Indian population: does Bolton's analysis apply? J Contemp Dent Pract 2014;15(1):103-7.

[5] Saritha T, Sunitha C, Kumar PK, et al. Applicability of Bolton's analysis to a South Telangana population. Indian Journal of Dental Sciences 2017;9(4):225-32.

[6] Patel YV, Nair VS, Jamenis SC. Bolton analysis of the Maratha population in Pune. Journal of Dental and Allied Sciences 2017;6(1):8-11.

[7] Lundstrom A. Intermaxillary tooth width ratio and tooth alignment and occlusion. Acta Odontol Scand 1955;12(34):265-92.

[8] Zachrisson BU. Improving orthodontic results in cases with maxillary incisors missing. Am J Orthod 1978;73(3):274-89.

[9] Thote AM, Uddanwadiker RV, Sharma K, et al. Optimum force system for intrusion and extrusion of maxillary central incisor in labial and lingual orthodontics. Computers in Biology and Medicine 2016;69:112-9.

[10] Baliga SM. Child oral health-care literacy in India: can access to services be improved? J Indian Soc Pedod Prev Dent 2019;37(1):1-2.

[11] Baliga S. Emerging trends in pediatric dentistry. J Indian Soc Pedod Prev Dent 2018;36(2):107. 